\title{
COMBINATION OF ACUPUNCTURE AND CHINESE MEDICINAL HERBS IN TREATING MODEL RATS WITH POLYCYSTIC OVARY SYNDROME
}

\section{Rui-Jie MA, ${ }^{1}$ Jue ZHOU, ${ }^{2}$ Jian-Qiao FANG, ${ }^{1}$ Dan-Hong YANG, ${ }^{1}$ and Fan $Q U^{3,4 *}$}

\author{
${ }^{1}$ The third clinical college of Zhejiang Chinese Medical University, Hangzhou, Zhejiang, China \\ ${ }^{2}$ College of Food Science and Biotechnology, Zhejiang Gongshang University, Hangzhou, Zhejiang, \\ China, \\ ${ }^{3}$ Women's Hospital, School of Medicine, Zhejiang University, Hangzhou, Zhejiang, China, \\ ${ }^{4}$ Pharmaceutical Sciences Division, King's College London, London SE1 9NH, UK \\ *E-mail: qufan43@yahoo.com.cn, fan.qu@kcl.ac.uk
}

\begin{abstract}
We explored the effects of combination of acupuncture and Chinese medicinal herbs in treating model rats with polycystic ovarian syndrome (PCOS) and to explore whether acupuncture has positive effects on the absorption of salvianolic acid B in the extracts of a Chinese medicine formula when treating the model rats. 60 female Sprague-Dawley (SD) rats were randomly divided into Groups A, B, C, D, E and F, with ten rats in each group. Except Group F, all of the other rats were induced to PCOS with oral administration of letrozole. The rats in Group F served as normal controls. Group A was treated with acupuncture. Group B was treated with oral administration of the extracts of the Chinese medicine formula. Group C was treated with a combination of oral administration of the extracts of Chinese medicine and acupuncture. Group D received western medicine as positive controls. After treatment, the serum levels of follicle stimulating hormone (FSH), luteinizing hormone( $\mathrm{LH}$ ) and testosterone ( $\mathrm{T}$ ) in each group were detected with the Enzyme-Linked ImmunoSorbent Assay (ELISA) and the serum concentration of salvianolic acid B were determined using High Performance Liquid Chromatography (HPLC). The serum levels of T and the ratio of LH/FSH in Group A, B. C, D, and F were significantly lower than those of Group E, indicating the model rats with PCOS were successfully established. Compared with Groups A, B, D and E, the serum levels of $\mathrm{T}$ and the ratio of LH/FSH in Group C were significantly lower respectively, indicating combination of acupuncture and Chinese medicinal herbs can significantly enhance curative effects in treating model rats with PCOS. The concentration of serum salvianolic acid Group C was significantly higher than Group B, indicating that acupuncture might improve the absorption of salvianolic acid B from the extracts of Salvia miltiorrhiza Bunge in the Chinese medicine formula. Combination of acupuncture and Chinese medicinal herbs significantly enhance curative effects in treating model rats with PCOS and acupuncture has positive effects in improving the absorption of salvianolic acid B in the extracts of the Chinese medicine formula when treating the model rats with PCOS.
\end{abstract}

Key words: Acupuncture; Chinese medicine; Polycystic ovarian syndrome (PCOS).

\section{Introduction}

Polycystic ovary syndrome (PCOS), as a heterogeneous endocrine disorder, with a prevalence of about $1 / 15$, is mainly characterized by ovarian hyperandrogenism, hyperinsulinemia from insulin resistance and reduced fecundity (Knochenhauer et al, 1998). PCOS is a complex endocrine and metabolic syndrome, which is characterized by small ovarian cysts, irregular menstrual cycles, abnormal hair growth, acne, and metabolic problems leading to weight gain (Knochenhauer et al, 1998). The cysts associated with PCOS cause a hormone imbalance by means of which sufferers have increased male sex hormones. This can disturb ovulation and lead to infertility. Women who suffer from PCOS also have a higher risk of 
developing hypertension and diabetes (Knochenhauer et al, 1998).

As early as two thousand years ago, clinical practitioners began to apply acupuncture and Chinese medicinal herbs together to treat various diseases in China (Ren, 1986). Acupuncture and Chinese medicinal herbs have both been found to significantly regulate the dysfunction of hypothalamic-pituitary-ovarian axis of the female (Chen,1997; Ren et al, 2005; Wu et al, 2008). As PCOS is associated with peripheral and central factors that influence sympathetic nerve activity, the sympathetic nervous system may be an important factor in the development and maintenance of PCOS (Stener-Victorin et al, 2008). Many women with PCOS may require prolonged treatment. Acupuncture has been found to affect PCOS via modulation of endogenous regulatory systems, including the sympathetic nervous system, the endocrine and the neuroendocrine system (Stener-Victorin et al, 2008). Many experiments in rat models of steroid-induced polycystic ovaries and clinical data from studies in PCOS patients suggest that acupuncture exert long-lasting positive effects on metabolic and endocrine systems and ovulation (Stener-Victorin et al, 2008).

However, few researches had explored the effects of combination of acupuncture and Chinese medicinal herbs in treating model rats with PCOS and to explore whether acupuncture has positive effects on the absorption of active compounds in the extracts of Chinese medicinal herbs when treating the model rats. The present study was then designated to explore the effects of combination of acupuncture and Chinese medicinal herbs in treating model rats with PCOS and to explore whether acupuncture has positive effects on the absorption of salvianolic acid B in the extracts of a Chinese medicine formula when treating the PCOS model rats.

\section{Materials and Methods}

\section{Preparation of the Extracts of the Chinese Formula}

The Chinese formula used to treat PCOS were composed with $30 \mathrm{~g}$ of Salvia miltiorrhiza Bunge, $35 \mathrm{~g}$ of Eucommia ulmoides Oliver, 20g of Carthamus tinctorius Linn., 20g of Bupleurum chinense DC., 30g of Gleditsia sinensis Lam. and $20 \mathrm{~g}$ of Atractylodes lancea( Thunb.)DC. The crude drugs of this formula were extracted by boiling water for $60 \mathrm{~min}$ to reach the final volume of $1550 \mathrm{ml}$. After filtration, the extraction procedure was repeated once to reach the final volume of $1240 \mathrm{~mL}$. All the filtrates were combined and condensed under reduced pressure and freeze dried. The flowchart of the extraction method is shown in Figure 1.

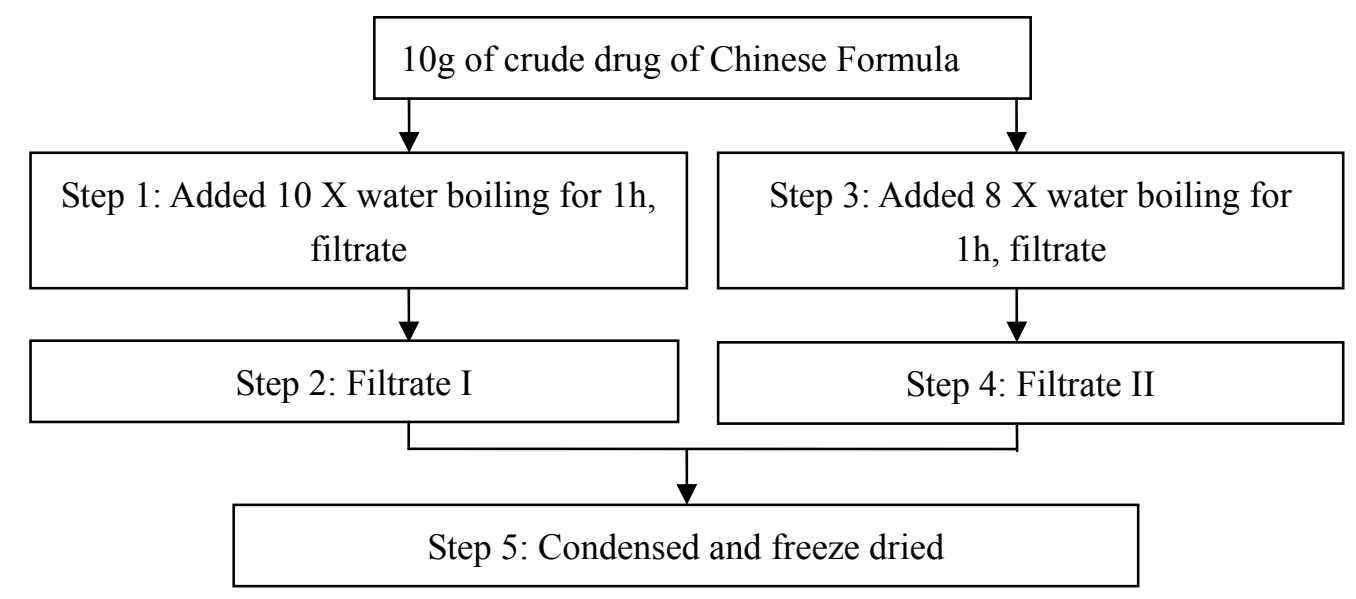

Figure 1: Preparation of the extracts of the Chinese Formula

Animals, groups and administration

60 female Sprague-Dawley (SD) rats (weighing 190土10g) were provided by the Laboratory Animal Center of Hangzhou Normal University (Hangzhou, China). The rats with two continuous sexual cycles were selected for this research. 
The research was carried out according to the National Research Council's protocol for the care and use of laboratory animals. The PCOS model was established by oral administration with letrozole dissolved in $0.5 \%$ of carboxymethyl cellulose (CMC, $2 \mathrm{ml} / \mathrm{kg}$ ) for 21 days once daily. 50 rats were orally administered with letrozole at the concentration of $1 \mathrm{mg} / \mathrm{kg}$ for 21 days. The 50 rats were randomly divided into five groups with 10 rats in each group to group A, group B, group C, group D and group E. 10 rats were randomly selected as group F received $0.5 \%$ of $\mathrm{CMC} 2 \mathrm{ml} / \mathrm{kg}$ body weight. The anovulatory PCOS model was confirmed successfully by the observation of the continuous keratinization of vaginal epithelium.

Group A: The rats in Group A were acupunctured at the following acupoints: Jizhong (Du6), Dazhui (Du14) and Zhongwan (Ren12). Both the acupoints and the stainless steel needles were sterilized with $75 \%$ alcohol. The rats were anesthetized with sodium pentobarbital (40 mg/kg, i.p.) before acupuncture treatment. The needles were inserted $2 \mathrm{~mm}$ into Jizhong (Du6), Dazhui (Du14) and Zhongwan (Ren12) and then were connected to an electro-therapeutic apparatus (Model G6805-2, Shanghai, China) for $15 \mathrm{~min}$ through clip electrodes. For the electronic stimulation, high-frequency $(100 \mathrm{~Hz})$ and low frequency $(20 \mathrm{~Hz})$ are used interchangeably with intensity ranged between $5 \mathrm{~mA}$ and $25 \mathrm{~mA}$. Slight twitches of the lower limbs of rats were found. The acupuncture treatment were taken every two days and continued for 28 days.

Group B: The rats in Group B were orally administered with the extracts of the Chinese medicine formula for 28 days dissolved in $0.5 \%$ of CMC with the dosage of $2.5 \mathrm{~g} / \mathrm{kg}$.

Group C: The rats were treated with a combination of oral administration of the extracts of the Chinese medicine formula $(2.5 \mathrm{~g} / \mathrm{kg})$ and acupuncture. $30 \mathrm{mins}$ after the oral administration of the extracts, the rats in Group C were acupunctured at the following acupoints: Jizhong (Du6), Dazhui (Du14) and Zhongwan (Ren12).

Group D: The rats were treated with oral administration of clomifene citrate dissolved in $0.5 \%$ of CMC with the dosage of $1 \mathrm{mg} / \mathrm{kg}$. Every four to five days make one sexual cycle of rats. At the first and second day of each cycle the rats were orally administered with clomifene citrate once per day. On the third to fifth day of the cycle, the oral administration was stopped. It was 28 days from the first day of oral administration.

Group E: The rats were treated with oral administration with $0.5 \%$ of CMC with the dosage of $1 \mathrm{mg} / \mathrm{kg}$.

Group F: The rats were treated with oral administration of $0.5 \%$ of CMC with the dosage of $1 \mathrm{mg} / \mathrm{kg}$.

From the Day 6 of the treatment, the vaginal smears of all the rats were observed to test ovulation. An hour after the treatments for 28 days on Day 50, all the rats in Groups A, B, C, D, E and F were anesthetized with sodium pentobarbital (40 $\mathrm{mg} / \mathrm{kg}$, i.p.). The blood samples were taken from hepatic portal vein for the analysis of follicle stimulating hormone (FSH), luteinizing hormone(LH) and testosterone (T) with Enzyme-Linked ImmunoSorbent Assay (ELISA). The blood samples were collected and centrifuged at 3,000 rpm for $10 \mathrm{~min}$. The supernatant serum samples were stored at $-20^{\circ} \mathrm{C}$ until analysis. The serum concentration of salvianolic acid B was determined using High Performance Liquid Chromatography (HPLC). The whole process of the animal treatment is shown in Figure 2.

\section{Materials}

Salvia miltiorrhiza Bunge was purchased from Huqing Yutang Pharmaceutical Co., Ltd (Hangzhou, China) and was identified by Zhejiang Chinese Medicinal University (Hangzhou, China). Salvianolic acid B was purchased from Carbosynth limited, UK. Acetonitrile of HPLC-grade was purchased from TEDIA Co. (OH, USA). Acetic acid and perchloric acid (analytical reagent grade) were purchased from Sinapharm Chemical Reagent Co. Ltd (Shanghai, China). Ultra-pure water (made by Millipore, Bedford, MA, USA) with a resistivity of more than $18 \mathrm{M} \Omega$ was used for all the preparations. The ELISA assay kits were provided by Boster Biotehnology Ltd., Wuhan, China.

\section{HPLC conditions}

Agilent 1200 SL (Agilent Technologies, Santa Clara, California, USA) was used in the research. The HPLC

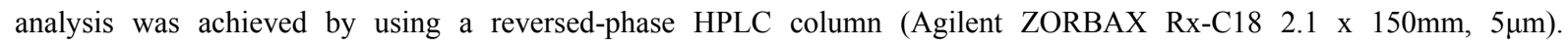
Water/acetic acid $(0.1 \% \mathrm{v} / \mathrm{v}, \mathrm{pH}=6.0$; used as solvent $\mathrm{A})$ and acetonitrile (as solvent $\mathrm{B}$ ) were used as mobile phase with a 
linear gradient elution at a flow rate of $0.25 \mathrm{~mL} / \mathrm{min}$. The linear gradient elution was as follows: solvent B was increased from 5 to $30 \%$ in the first $25 \mathrm{~min}$, then increased to $35 \%$ till $40 \mathrm{~min}$, increased to $75 \%$ till $60 \mathrm{~min}$ and then increased to $85 \%$ at $70 \mathrm{~min}$. The sample injection volume was $20 \mu \mathrm{l}$. The detection wavelength was set at $280 \mathrm{~nm}$. The column temperature was $25^{\circ} \mathrm{C}$. The solvents were filtered through a $0.45 \mu \mathrm{m}$ Millipore filter and degassed prior to use.

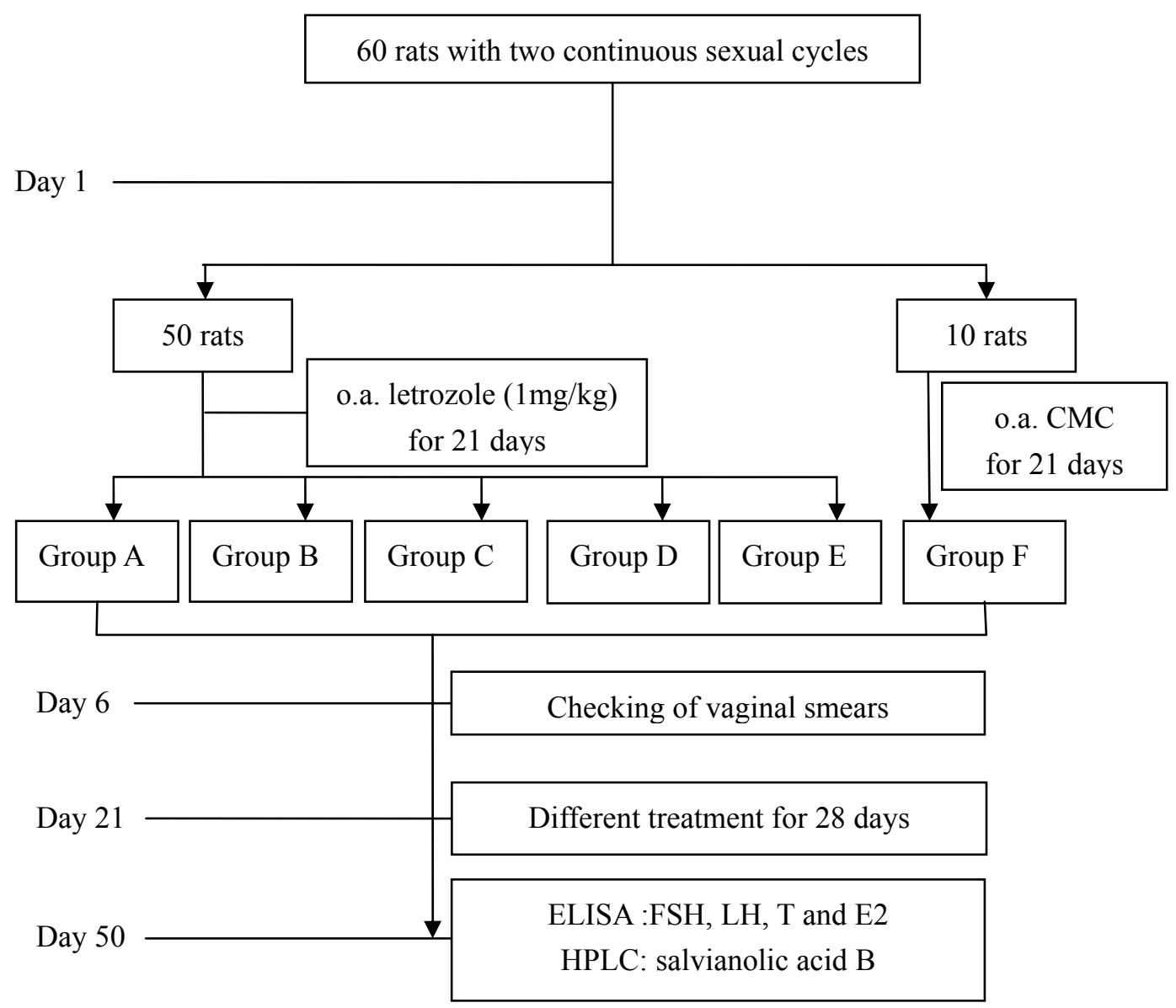

Figure 2: The whole process of the research

\section{Preparation of stock and working standard solutions}

The standard stock solution was prepared by dissolving $0.69 \mathrm{mg}$ of salvianolic acid $\mathrm{B}$ in $10 \mathrm{~mL}$ of methanol to obtain a concentration of $69.00 \mu \mathrm{g} / \mathrm{mL}$. The stock solution was kept at $4^{\circ} \mathrm{C}$. The standard working solutions were prepared at various concentrations of $0.01,0.07,0.69,6.90$ and $69.00 \mu \mathrm{g} / \mathrm{mL}$ by diluting the stock solution with methanol.

\section{Preparation of the samples}

$0.2 \mathrm{~mL}$ of methol solution was added into $1 \mathrm{~mL}$ of serum. The mixture was then vortex-mixed for 2 mins. After centrifugation at 3,000 rpm for 10 mins, the supernatant was filtered through a $0.45 \mu \mathrm{m}$ membrane filter unit. $20 \mu \mathrm{L}$ of each sample solution was analyzed by HPLC. 


\section{Preparation of calibration standards}

The samples for standard calibration curve were prepared by spiking $100 \mu$ of the blank rat serum with $100 \mu \mathrm{L}$ of the appropriate working solutions to yield the concentrations of $0.01,0.04,0.35,3.45$ and $34.50 \mu \mathrm{g} / \mathrm{mL}$. The calibration curve was plotted with the concentrations as $\mathrm{X}$-axes and the peak areas as Y-axes using linear regression.

\section{Validation of the methods}

The samples for calibration curve $(n=5)$ were prepared in the blank serum according to the methods of preparing the calibration standards. A correlation coefficient of more than 0.99 was desirable for the calibration curve. Recovery was determined by adding salvianolic acid B of different concentrations: $0.06,0.59$ and $5.90 \mu \mathrm{g} / \mathrm{mL}$ respectively. The precision (intra-day and inter-day) of the methods was also calculated at the three concentrations above. The precision assay was determined by the variability of the peak areas at each concentration. The accuracy was determined by measuring of concentration with the spiked value. The limit of detection (LOD) was determined at a signal-to-noise of baseline at a ratio of $3: 1$.

\section{ELISA analysis}

The serum levels of FSH, LH, and T were analyzed using ELISA kits according to the instruction.

\section{Statistical analysis}

Results were analyzed by an independent university-statistician using the computer software, Statistical Package for Social Sciences (SPSS 13.0 for Windows). Analysis of variance (ANOVA) was used for comparison of the serum concentration of salvianolic acid B and for comparison of the serum levels of FSH, LH, and T. For all hypothesis tests, a 5\% significance level $\mathrm{p}<0.05$ ) and two-tailed tests were used.

\section{Results}

Comparison of Serum FSH, LH and T levels and the ratio of LH/FSH.

As shown in Figure 3, the serum levels of T and the ratio of LH/FSH in Group A, B. C, D, and F were significantly lower than those of Group E, indicating that the model rats with PCOS were successfully established. Compared with Groups $\mathrm{A}, \mathrm{B}, \mathrm{D}$ and $\mathrm{E}$, the serum levels of $\mathrm{T}$ and the ratio of LH/FSH in Group C were significantly lower respectively, indicating combination of acupuncture and Chinese medicinal herbs can significantly enhance curative effects in treating model rats with PCOS.

\section{Validation of the method}

The regression equations and correlation coefficients $\left(R^{2}\right)$ of salvianolic acid B were derived as $\mathrm{Y}=888216 \mathrm{X}-1957$ $\left(R^{2}=0.99\right)$. The linear range of the concentration of salvianolic acid B was from 0.01 to $34.50 \mu \mathrm{g} / \mathrm{mL}$. The standard curves were obtained with the peak area of standards as the $\mathrm{Y}$-axis and the salvianolic acid $\mathrm{B}$ concentration as the $\mathrm{X}$-axis. The intra-day accuracy ranged from 121.05 to $148.50 \%$ ( $\mathrm{RSD}<10 \%$ ) and the inter-day accuracy ranged from 119.13 to $142.66 \%$ $(\mathrm{RSD}<10 \%)$. The LOD of salvianolic acid B was between $5 \mathrm{ng}$ and $10 \mathrm{ng}$. The concentration of salvianolic acid B was respectively $0.06,0.59$ and $5.90 \mu \mathrm{g} / \mathrm{mL}$. 


\section{Analysis of the samples}

The HPLC chromatogram of a Salvia miltiorrhiza Bunge is shown in Figure 3. The peaks at 30.052 min is salvianolic acid

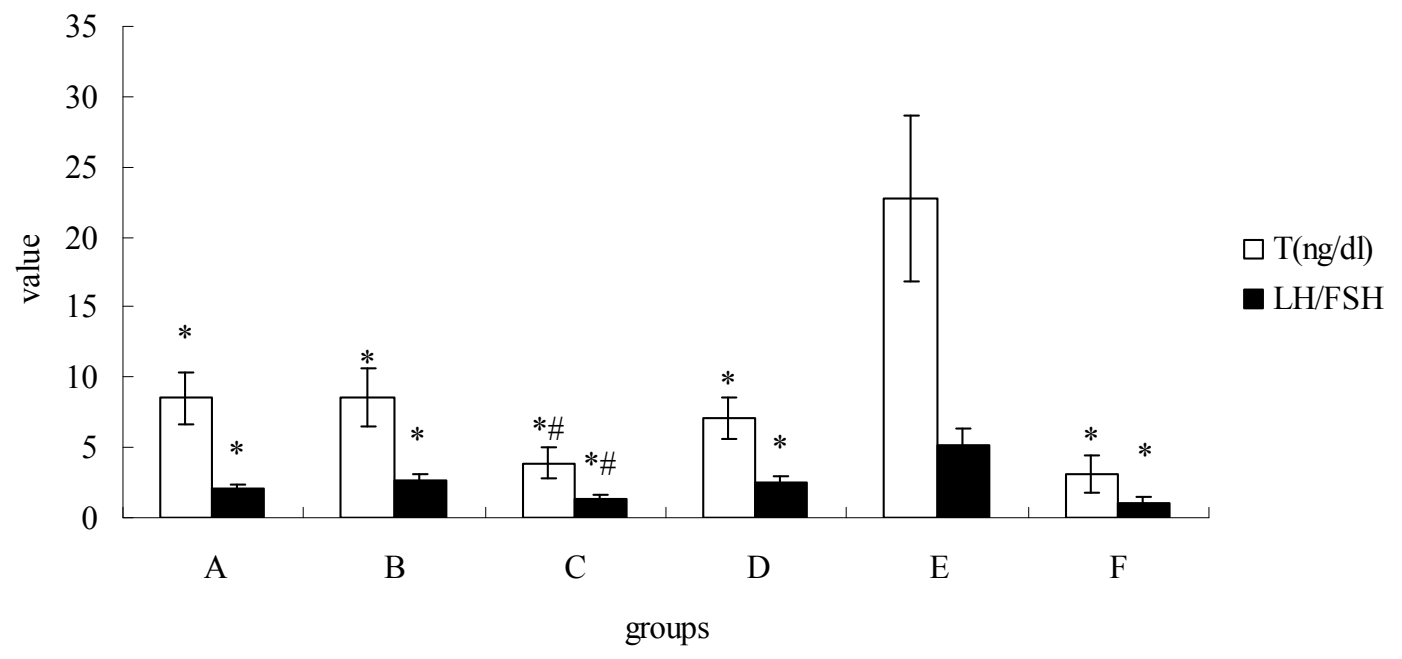

Note: * $\mathrm{P}<0.05$, compared with Group E; $\quad{ }^{\#} \mathrm{P}<0.05$, Group C compared with Group D

Figure 3: Comparison of Serum FSH, LH and T levels and the ratio of LH/FSH.

B.

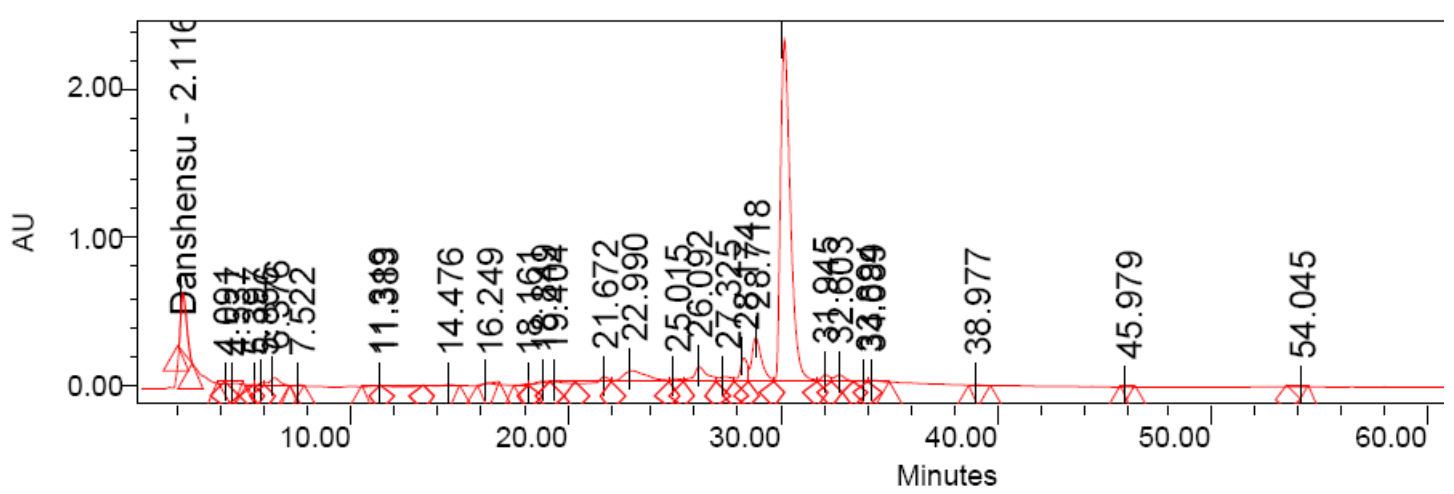

Figure 4: HPLC chromatogram of Salvia miltiorrhiza Bunge samples

The concentration of the serum salvianolic acid B is shown in Table 2. The serum concentration of salvianolic acid B in group B was $1.65 \pm 0.36 \mu \mathrm{g} / \mathrm{mL}$. The peak serum concentrations of salvianolic acid B in group C was $2.52 \pm 0.49 \mu \mathrm{g} / \mathrm{mL}$. The concentration of serum salvianolic acid Group C was significantly higher than Group B, indicating that acupuncture might improve the absorption of salvianolic acid B from the extracts of Salvia miltiorrhiza Bunge in the rats.

Table 1: Comparison of the body weight and serum salvianolic acid B between group B and C

\begin{tabular}{cccc}
\hline Group & $\mathrm{n}$ & Weight $(\mathrm{g})$ & Concentration $(\mu \mathrm{g} / \mathrm{mL})$ \\
\hline B & 6 & $191.1 \pm 8.5$ & $1.65 \pm 0.36$ \\
C & 6 & $190.9 \pm 7.5$ & $2.52 \pm 0.49^{*}$ \\
\hline
\end{tabular}

Note: $* \mathrm{P}<0.05$, compared with group $\mathrm{B}$. 


\section{Discussion}

According to the theory of Traditional Chinese medicine, PCOS belongs to the category of both of an excess condition (such as Phlegm Dampness and Liver-Qi Excess) and a deficient condition (such as Kidney Yin/Yang Deficiency) (Chen,1997; Ren et al, 2005; Wu et al, 2008). As acupuncture and Chinese medicinal herbs can be served as natural approaches to help strengthen and regulate the hormonal systems of the female body, encourage conception and a healthy pregnancy, they can be used as a safe, effective, and natural whole body approach to treat PCOS (Chen,1997; Ren et al, 2005; Wu et al, 2008).

In recent years, many clinical and animal experiments have been conducted to explore the effects of acupuncture on PCOS. In a recent clinical study (Jedel et al, 2010), the researchers randomized 84 women with PCOS, aged 18-37 years, to 16 weeks of low-frequency electro-acupuncture, physical exercise, or no intervention. The primary outcome measure changes in the concentration of total $\mathrm{T}$ at week 16 determined by gas and liquid chromatography-mass spectrometry was analyzed by intention to treat. Secondary outcome measures were changes in menstrual frequency; concentrations of androgens, estrogens, androgen precursors, and glucuronidated androgen metabolites; and acne and hirsutism. Outcomes were assessed at baseline, after 16 weeks of intervention, and after a 16-week follow-up. After 16 weeks of intervention, circulating $\mathrm{T}$ decreased by $-25 \%$, androsterone glucuronide by $-30 \%$, and androstane- $3 \alpha, 17 \beta$-diol-3-glucuronide by $-28 \%$ in the electro-acupuncture group ( $\mathrm{P}=0.038,0.030$, and 0.047 , respectively vs. exercise); menstrual frequency increased to 0.69 /month from 0.28 at baseline in the electro-acupuncture group ( $\mathrm{P}=0.018$ vs. exercise). After the 16-week follow-up, the acne score decreased by $-32 \%$ in the electro-acupuncture group ( $\mathrm{P}=0.006$ vs. exercise). Both electro-acupuncture and exercise improved menstrual frequency and decreased the levels of several sex steroids at week 16 and at the 16-week follow-up compared with no intervention. Low-frequency electro-acupuncture and physical exercise improved hyperandrogenism and menstrual frequency more effectively than no intervention in women with PCOS. They finally found that low-frequency electro-acupuncture was superior to physical exercise and may be useful for treating hyperandrogenism and oligo/amenorrhea. A recent animal experiment (Johansson et al, 2010) was designated to test the hypothesis that low-frequency electro-acupuncture regulate mediators involved in skeletal muscle glucose uptake and metabolism and alters the lipid profile in rats with dihydrotestosterone (DHT)-induced PCOS. To test this hypothesis, they implanted in prepubescent female rats 90-day continuous-release pellets containing DHT. At 70 days of age, the rats were randomly subdivided into two groups: one received low-frequency electro-acupuncture (evoking muscle twitches) for 20-25 min five times/week for 4-5 week; while the other did not. Controls were implanted with pellets containing vehicle only. All three groups were otherwise handled similarly. Lipid profile was measured in fasting blood samples. Insulin sensitivity was determined by euglycemic hyperinsulinemic clamp, soleus muscle protein expression of glucose transporter 4 (GLUT4), and phosphorylated and nonphosphorylated Akt, and Akt substrate of $160 \mathrm{kDa}$ was determined by Western blot analysis and GLUT4 location by immunofluorescence staining. PCOS electro-acupuncture rats had normalized insulin sensitivity, lower levels of total high-density lipoprotein and low-density lipoprotein cholesterol, and increased expression of GLUT4 in different compartments of skeletal muscle compared with PCOS rats. Total weight and body composition did not differ in the groups. Thus, in rats with DHT-induced PCOS, low-frequency electro-acupuncture has systemic and local effects involving intracellular signaling pathways in muscle that may, at least in part, account for the marked improved insulin sensitivity.

The present research found that the serum levels of $\mathrm{T}$ and the ratio of LH/FSH in Group A, B. C, D, and F were significantly lower than those of Group E, indicating the model rats with PCOS were successfully established. Compared with Groups A, B, D and E, the serum levels of T and the ratio of LH/FSH in Group C were significantly lower respectively, indicating combination of acupuncture and Chinese medicinal herbs can significantly enhance curative effects in treating model rats with PCOS. The concentration of serum salvianolic acid Group C was significantly higher than Group B, indicating that acupuncture might improve the absorption of salvianolic acid B from the extracts of the Chinese medicine formula in the PCOS rats.

Both of acupuncture and Chinese medicinal herbs have significant advantages in treating anovulation caused by 
PCOS. In a clinical research (Stener-Victorin et al, 2000) aiming to evaluate if electro-acupuncture could affect oligo-/anovulation and related endocrine and neuroendocrine parameters in women with PCOS, 24 women (between the ages of 24 and 40 years) with PCOS and oligo-/amenorrhea were included in this non-randomized, longitudinal, prospective study. The study period was defined as the period extending from 3 months before the first electro-acupuncture treatment, to 3 months after the last electro-acupuncture treatment (10-14 treatments), in 8-9 months. The menstrual and ovulation patterns were confirmed by recording of vaginal bleedings and by daily registrations of the basal body temperature (BBT). Blood samples were collected within a week before the first electro-acupuncture, within a week after the last electro-acupuncture and 3 months after electro-acupuncture. The results showed that $38 \%$ of the women experienced a good effect. They displayed a mean of 0.66 ovulations/woman and month in the period during and after the electro-acupuncture period compared to a mean of 0.15 before the electro-acupuncture period $(\mathrm{p}=0.004)$. Before electro-acupuncture, women with a good effect had a significantly lower body-mass index (BMI) $(\mathrm{p}<0.001)$, waist-to-hip circumference ratio (WHR) $(\mathrm{p}=0.0058)$, serum testosterone concentration $(\mathrm{p}=0.0098)$, serum testosterone/sex hormone binding globulin (SHBG) ratio $(\mathrm{p}=0.011)$ and serum basal insulin concentration $(\mathrm{p}=0.0054)$, and a significantly higher concentration of serum SHBG $(\mathrm{p}=0.040)$ than did those women with no effect. It was the concluded that the repeated electro-acupuncture treatments induce regular ovulations in more than one third of the women with PCOS. The group of women with good effect had a less androgenic hormonal profile before treatment and a less pronounced metabolic disturbance compared with the group with no effect. For this selected group, electro-acupuncture offers an alternative to pharmacological ovulation induction. Together with changes in diet and rational exercise, use of the combination of acupuncture and Chinese medicinal herbs are supposed to empower PCOS women to lead a better quality of lives.

\section{Conclusion}

Combination of acupuncture and Chinese medicinal herbs significantly enhanced curative effects in treating model rats with PCOS and acupuncture had positive effects in improving the absorption of salvianolic acid B in the extracts of the Chinese medicine formula when treating the model rats with PCOS.

\section{Acknowledgements}

The Project was supported by Open Research Fund of Zhejiang First-foremost Key Subject-Acupuncture \& Moxibustion (No.ZTK 2010A 04).and Zhejiang Traditional Chinese Medicine Foundation (No. 2008YB010).

\section{References}

1. Chen, B.Y., (1997). Acupuncture normalizes dysfunction of H-P-O axis. Acupunct Electrother Res 22:97-108

2. Jedel, E., Labrie, F., Odén, A., Holm, G., Nilsson, L., Janson, P.O., Lind, A.K., Ohlsson, C., Stener-Victorin, E.,(2010) Impact of electro-acupuncture and physical exercise on hyperandrogenism and oligo/amenorrhea in women with polycystic ovary syndrome: a randomized controlled trial. Am J Physiol Endocrinol Metab 300:E37-45.

3. Johansson, J., Feng, Y., Shao, R., Lönn, M., Billig, H., Stener-Victorin, E.,(2010) Intense electroacupuncture normalizes insulin sensitivity, increases muscle GLUT4 content, and improves lipid profile in a rat model of polycystic ovary syndrome. Am J Physiol Endocrinol Metab 299:E551-559.

4. Knochenhauer, E.S., Key, T.J., Kahsar-Miller, M., Waggoner, W, Boots, L.R., Azziz, R., (1998). Prevalence of the polycystic ovary syndrome in unselected black and white women of the southeastern United States: a prospective study. J Clin Endocrin Metab 83:3078-3082

5. Ren, Q.L., Tan, Y., (2005). Treating PCOS with Chinese medicinal herbs: A review. Liaoning JTCM 32: 17-19 
Ma et al., Afr J Tradit Complement Altern Med. (2011) 8(4):353 - 361

6. Ren, Y.Q., (1986). Index of Huangdi Neijing (The Yellow Emperor's Classic of Internal Medicine). Beijing: People's Medical Publishing House 97-134

7. Stener-Victorin, E., Waldenström, U., Tägnfors, U., Lundeberg, T., Lindstedt, G., Janson, P.O.,(2000) Effects of electro-acupuncture on anovulation in women with polycystic ovary syndrome. Acta Obstet Gynecol Scand. 79:180-188.

8. Stener-Victorin, E., Jedel, E., Mannerås, L., (2008). Acupuncture in polycystic ovary syndrome: current experimental and clinical evidence. J Neuroendocrinol. 20:290-298

9. Wu, X., Yang, Z.W., (2009).Treatment of PCOS With Traditional Chinese and Western Medicine. Aca J Chin Med 15:111-113 